\title{
Morfometría de la subcuenca del río Toyogres, Cartago, Costa Rica
}

\author{
Morphometry of Toyogres river sub-basin, Cartago, Costa Rica
}

\author{
Pablo Ramirez-Granados ${ }^{1}$ \\ Kevin Alfaro-Jiménez ${ }^{2}$ \\ Universidad Nacional de Costa Rica
}

http://dx.doi.org/10.15359/rgac.60-1.9

\begin{abstract}
RESUMEN
A partir de sistemas de información geográfica y modelado de datos en Microsoft Excel, se realizó la morfometría de la subcuenca del río Toyogres en Cartago. El objetivo del estudio fue desarrollar los principales parámetros geomorfométricos y morfométricos que caracterizan una cuenca hidrográfica como etapa inicial en cualquier estudio hidrológico. Los resultados mostraron como la forma de la subcuenca puede ser analizada a partir del estudio de los aspectos geométricos derivados de los índices morfomrétricos, los cuales a su vez relacionan los aspectos litológicos y geomorfológicos que condicionan la forma alargada que esta tiene. A su vez, esta forma condiciona la relación de sus drenajes y las categorías en las cuales se encuentran además de su respuesta hidrológica.
\end{abstract}

Palabras clave: morfometría, cuenca Toyogres,

1 Investigadores, Laboratorio de Hidrogeología y Manejo de Recursos Hídricos, Escuela de Ciencias Ambientales, Universidad Nacional de Costa Rica. Correo electrónico: pablo.ramirez.granados@una.cr

2 Investigadores, Laboratorio de Hidrogeología y Manejo de Recursos Hídricos, Escuela de Ciencias Ambientales, Universidad Nacional de Costa Rica. Correo electrónico: kevin15aj@gmail.com

Fecha de recepción: 31 de mayo de 2017

Fecha de aceptación: 4 de setiembre de 2017 


\begin{abstract}
Based on geographic information systems and data modeling in Microsoft Excel, the morphometry of the Toyogres river sub-basin in Cartago was modeled. The purpose of the study was to develop the main geomorphometric and morphometric parameters that characterize a hydrographic basin as an initial stage in any hydrological study. The results showed how the shape of the sub-basin is related to the geometric aspects derived from the indices, which, therefore, responds to an elongated form. In turn, this form conditions the relationship of their drainage and the categories in which they are located.
\end{abstract}

Keywords: morphometry, Toyogres River, watershed.

\title{
Introducción
}

El manejo de los recursos hídricos es, en la actualidad una de las mayores preocupaciones de la humanidad debido a las necesidades de agua para su consumo y las diferentes actividades humanas. Una de las maneras para abordar la problemática de los recursos hídricos, es a partir del enfoque de cuenca hidrográfica, el cual permite conocer las características intrínsecas y la hidrología (Brooks et al., 2013).

En la evaluación inicial de una cuenca hidrográfica, una de las primeras etapas lo constituye la medición de ella, basada en parámetros morfométricos (Zăvoianu, 1985). La morfometría de cuencas es un término que se refiere a la medición de la forma de estas (Zaidi 2011; Esper Angillieri y Perucca 2014) y como la forma condiciona su respuesta hidrológica. También puede ser usada para entender la dinámica geomorfológica de un paisaje (Demoulin, 2011).

Las cuencas hidrográficas o cuencas de drenaje son las unidades fundamentales para entender las características geométricas de paisajes fluviales, tales como la topología de redes de corrientes, su patrón, forma y características de relieve (Prabu y Baskaran, 2013; Mikhailova, 2015). Por otro lado, la estimación de los parámetros y patrones de drenaje, a partir de la morfometría, pueden contribuir a un mejor entendimiento del comportamiento hidrológico de una cuenca, como por ejemplo el estudio de inundaciones (Newson 1978), la ocurrencia, movimiento y almacenamiento de agua subterránea (Avinash et al. 2014)

El presente trabajo representa una descripción morfométrica inicial de la subcuenca hidrográfica del río Toyogres. Constituye un estudio inicial, a partir del cual se podrán realizar propuestas en relación al comportamiento hidrológico y las propiedades morfológicas de la cuenca, 
integrando principios definidos en hidrología, geomorfología y otras disciplinas (Shen et al. 2015). Este artículo es un resultado parcial del proyecto 0138-16 denominado "Caracterización biofísica, morfométrica e hidrológica para proponer acciones de manejo de recursos hídricos en la subcuenca del río Páez, Cartago" y constituye uno de los aportes en investigación técnica y científica aplicada, del Laboratorio de Hidrogeología y Manejo de Recursos Hídricos, de la Escuela de Ciencias Ambientales, a la Comisión de Recurso Hídrico del Consejo Cantonal de Coordinación Institucional del Cantón de Cartago (CCCI-RH).

\section{Área de Estudio}

El área de la cuenca se localiza en la zona central de la hoja Istarú, desde el poblado de Tierra Blanca, atravesando la ciudad de Cartago, hasta llegar al poblado de Dulce Nombre (fig. 1). Se ubica entre las coordenadas planas 508770 y $512130 \mathrm{~m}$ este y 1087483 y $1097263 \mathrm{~m}$ norte del sistema de Proyección Transversal de Mercator para Costa Rica (CRTM).

Posee un área aproximada de $9,8 \mathrm{~km}^{2}$, a partir de su proyección horizontal sobre contornos topográficos de la hoja Istarú, escala 1: 50.000 del Instituto Geográfico Nacional (IGN), usando un Sistema de Información Geográfica (SIG). Sus aguas drenan hacia el río Agua Caliente, el cual a su vez desemboca en el río Grande de Orosi para formar el río Reventazón. Dentro de la cuenca se destacan la ciudad de Cartago y los poblados de Tierra Blanca y Dulce Nombre. 


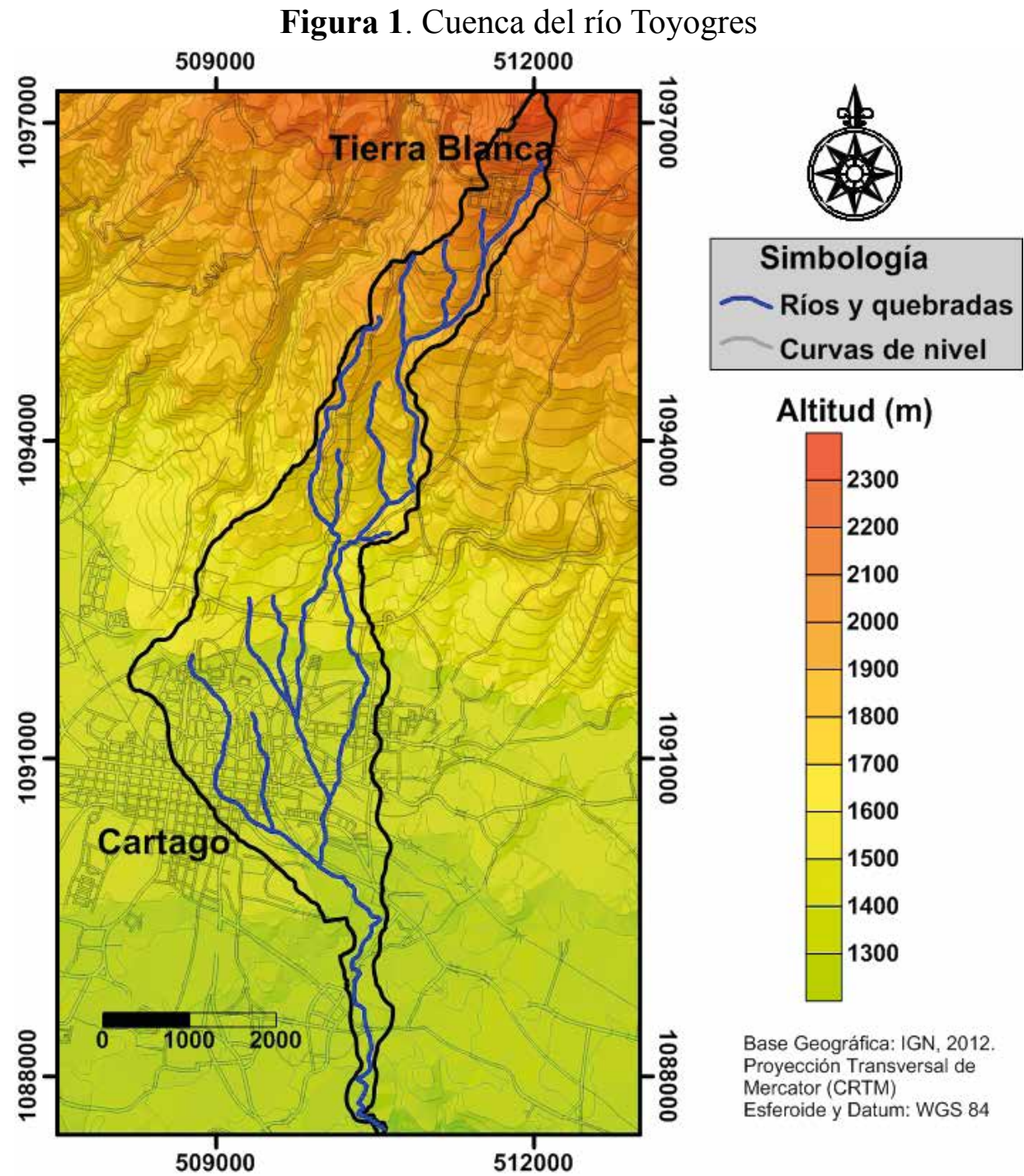

En la parte alta de la cuenca del río Toyogres, correspondiente al sector norte de la hoja Istarú, está conformada por un uso de la tierra predominantemente de cultivo agrícola anual, mientras que la parte sur, corresponde a zona urbana (Méndez, 2012).

Regionalmente en la cuenca se definen varias unidades geológicas correspondientes al Grupo Irazú, el cual se define como una serie de rocas 
volcánicas, producto de la actividad del volcán Irazú y que abarca desde el Pleistoceno Tardío hasta la actualidad (Krushensky, 1972). Krushensky (1972) divide este grupo en orden cronológico en las formaciones Reventado Sapper, Birrís y Cervantes. Posteriormente Alvarado (1993) propone las siguientes unidades en orden estratigráfico: Ignimbrita, Reventado, De La Haya, Cerro Alto Grande, Sapper, Birrís, Colada de Cervantes, González, Dóndoli, Tristán, Alfaro y Depósitos Recientes. Luego, Pavanelli et al. (2004) realizaron un estudio en las faldas sur-sureste, definiendo como unidades estratigráficas las siguientes: Indiferenciada, Cot, Debris Avalanche de Birrís, Flujos de Lava de Cervantes, Ceniza de Caída Laguna, Debris Avalanche de Reventado y Productos Recientes. Más reciente, Alvarado et al. (2006), proponen un nuevo orden estratigráfico para las unidades volcanológicas, definiendo las siguientes: San Jerónimo, Paraíso y Pico de Piedra, Reventado, De La Haya, Sapper, Cervantes, González, Dóndoli, Tristán, Alfaro y Depósitos Recientes. Para las descripciones de los materiales de la subcuenca del río Toyogres, se utilizarán las unidades definidas por Krushensky (1972), pues las descripciones de los trabajos posteriores obedecen a una separación de orden volcanológico.

La unidad geológica regional donde se encuentra la subcuenca del río Toyogres, corresponde con el Miembro Superior de la Formación Reventado. Esta consta de flujos de lava de tipo andesítico-basáltico, lahares y depósitos de ceniza. Localmente se encuentra compuesta por materiales volcánicos cuaternarios (Quintanilla et al., 2008) específicamente coluvios, lahares y aluviones. Estos lahares se caracterizan por presentar varios niveles de depositación separados por lentes arcillosos. Más recientemente, Ramírez y Zúñiga (2014) encontraron que estos lahares están sobreyacidos por capas de arcilla en distintos sectores. En el sector de Tierra Blanca, se reconocen flujo de lavas, mineralógicamente compuestas por cristales de plagioclasa en su mayoría, con un pequeño porcentaje de piroxenos y cuyo afloramiento se encuentra aproximadamente $1 \mathrm{~km}$ al noroeste de Tierra Blanca. Estas lavas están sobreyacidas por capas de ceniza, las cuales condicionan la forma ondulada suave del terreno (Ramírez-Granados y Zúñiga Mora 2014).

Con respecto a la geomorfología, Alvarado et al. (2006) señalan que el patrón de drenaje de los ríos al sur del volcán Irazú, fluye hacia abajo formando profundos cañones, debido al material volcánico poco 
consolidado. Algunos ejemplos de ello se manifiestan en el cañón del río Toyogres, en el puente que divide San Rafael de Oreamuno y Cartago, la carretera hacia el volcán Irazú y en la carretera hacia Paraíso; también se observan en la localidad de Dulce Nombre (Ramírez y Zúñiga, 2014).

Considerando los usos de la tierra definidos y cartografiados por PRUGAM (2008), los usos dominantes en esta cuenca señalan un alta dominancia de los pastos y los cultivos, desde la parte media de la cuenca hasta su cabecera, cerca del poblado de Tierra Blanca. De la cuenca media hacia el sur, los usos dominantes son el uso urbano y en mucho menor medida los pastos, muchos de los cuales presentan diferencias significativas con los pastos de la parte alta de la cuenca debido al impacto que se generan en ellos por los usos urbanos vecindantes.

\section{Metodología}

La elaboración de la morfometría de la cuenca se baso en las curvas topográficas de la hoja Istarú, escala 1:50 000, del Instituto Geográfico Nacional (IGN). A partir de la hoja previamente escaneada y georeferenciada y usando el sistema de información geográfica (SIG), ArcGis 10.2, se realizó la digitalización de los drenajes y el parteaguas, usando los contornos de elevación y con ello se trazó el límite topográfico de la cuenca hidrográfica. El modelado geomorfométrico se realizó con la ayuda del software Surfer 11.6.

Tomando en cuenca que la cuenca hidrográfica es un vector poligonal, se obtuvieron de ella sus propiedades geométricas: área y perímetro. Sobreponiendo el vector poligonal de líneas de contornos topográficos con el vector poligonal de la cuenca, se hizo un corte del polígono basado en las curvas de nivel y de allí se obtuvieron las áreas parciales de la cuenca entre contornos. En el caso de los vectores lineales, correspondientes a ríos y curvas de nivel, se obtuvieron las longitudes de línea para cada uno.

Ya obtenidos los insumos vectoriales en el sistema SIG, los parámetros morfométricos fueron calculados considerando la definición teórica de ellos, referida en distintas publicaciones (Zăvoianu, 1985; Villón, 2004; Vittala et al., 2004) y realizando diversos cálculos con ayuda de Microsoft Excel 2007.

En lo que respecta al modelado geomorfométrico, este se realizó utilizando una interpolación lineal de un conjunto de puntos espaciados 
$10 \mathrm{~m}$, con lo cual se construyó el modelo digital del terreno (MDT) sobre el cual se calcularon los parámetros morfométricos. Los parámetros geomorfométricos, expresados como ecuaciones diferenciales en derivadas parciales, fueron estimados usando una grilla cuadrada y utilizando diferencias finitas (Moore et al., 1991).

\section{Resultados}

\section{Pendiente}

Este parámetro geomorfométrico, calcula la pendiente de cualquier nodo de cuadrícula en la superficie. Esta pendiente es reportada en grados desde $0^{\circ}$ (horizontal) a $90^{\circ}$ (vertical). Para un punto particular en la superficie, la pendiente está basada en la dirección de descenso o ascenso más empinado en ese punto (Jordan, 2007) y es descrita por la siguiente ecuación:

$$
S=\sqrt{\left(\frac{\partial L}{\partial x}\right)^{2}+\left(\frac{\partial L}{\partial x}\right)^{2}}
$$

Para la cuenca del río Toyogres, las pendientes rondan valores entre los $0^{\circ}$ y $10^{\circ}$ en las zonas más planas de la cuenca baja, se incrementan hasta en $35^{\circ}$ en la zona media y alta (fig. 2). Uno de las características más evidentes en el mapa de pendientes, corroborado en la visitas de campo, es que los drenajes de la cuenca no manifiestan cañones profundos y muchos de ellos no son evidentes en contornos cada $20 \mathrm{~m}$. En varios de los trayectos a los largo del río, se observaron profundidades variables de hasta $5 \mathrm{~m}$, como en el caso del puente del río, sobre la vía 219, entre Cartago y Oreamuno, y los puentes en San Rafael de Oreamuno accesibles por los caminos principales. 
Figura 2. Pendiente $\left(^{\circ}\right)$ de la cuenca del río Toyogres.

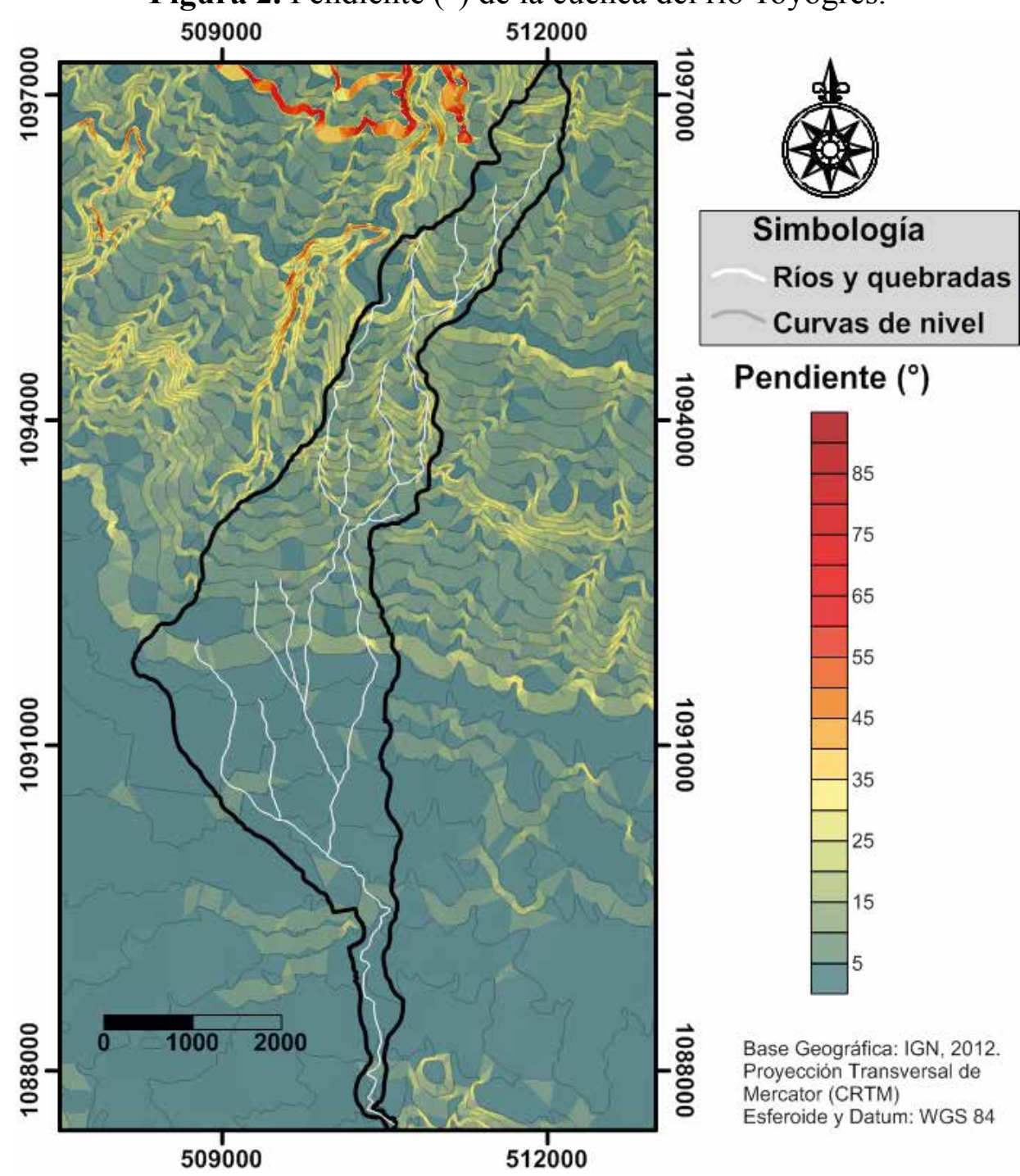

\section{Orientación}

La orientación calcula la dirección de descenso de la pendiente más empinada, es decir la dirección de inmersión, en cada nodo de cuadrícula. Esta es la dirección que aparece perpendicular a las líneas de contorno en la superficie y está opuesta a la dirección del gradiente. Los valores de 
Pablo Ramírez-Granados, Kevin Alfaro-Jiménez. Morfometría de la subcuenca del río Toyogres, Cartago, Costa Rica

orientación son reportados en acimut, donde $0^{\circ}$ señala una dirección norte y las sucesivas en relación horaria. Se describe como:

$$
A_{T}=270-\frac{360}{2 \pi} \cdot \operatorname{atan} \cdot 2\left(\frac{\partial L}{\partial y}, \frac{\partial L}{\partial x}\right)
$$

La orientación de las pendientes en la cuenca del rio Toyogres, muestra una dominancia en la dirección en la dirección sur y suroeste, especialmente en la cuenca media. Hacia el norte de la cuenca, los drenajes evidencian una clara división entre las pendientes, orientadas hacia el sur oeste y hacia el sureste. En las zonas donde se indefine la orientación, es decir, las zonas no orientadas, estas se caracterizan por ser zonas planas. En el campo estas zonas corresponden con el sector norte de la ciudad de Cartago, en el poblado de San Blas, donde la pendiente es baja. 
Figura 3. Orientación $\left(^{\circ}\right)$ de la cuenca del río Toyogres

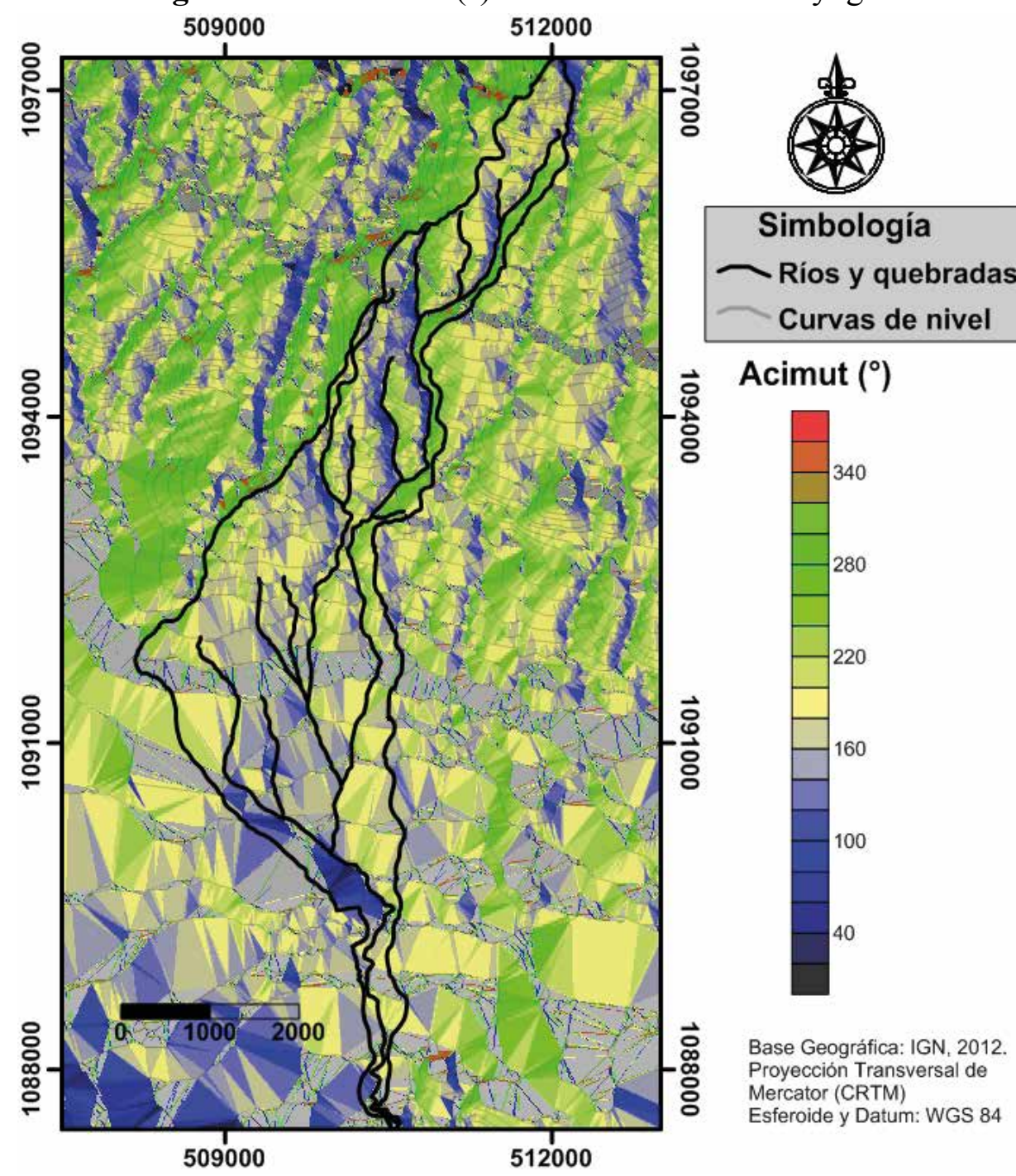

Geometría básica

La cuenca de captación del río Toyogres presenta un área de 10,14 $\mathrm{km}^{2}$, obtenida a partir de la digitalización de su parteaguas en un sistema de información geográfica. Según la definición de jerarquías, esta cuenca se puede considerar dentro de la categoría de minicuenca ya que su área 
está dentro del rango de 10 a $30 \mathrm{~km}^{2}$ (Vittala y Govindaiah 2004), para este trabajo su jerarquía corresponde a la de subcuenca.

Presenta un perímetro de 25,27 km y cuenta con una elevación mínima de $1280 \mathrm{~m}$ y una máxima de $2280 \mathrm{~m}$.

El parteaguas de una cuenca, define la forma y la superficie que abarca la cuenca hidrográfica, además influye en los escurrimientos y en la relación de caudal-tiempo (hidrograma) en el punto de salida. Los escurrimientos a los sistemas de drenajes, están condicionados por el contorno, de la misma manera que el tiempo de concentración. Para la cuenca del río Toyogres, su índice de compacidad es de 2,22, indicando que es una cuenca de forma alargada (Zăvoianu, 1985).

La longitud de una cuenca es definida como la longitud a lo largo de la dimensión proyectada más grande en lo posible paralela al drenaje principal ( $\mathrm{Pa}-$ reta y Pareta, 2011). La cuenca del río Toyogres, presenta una longitud de 10,18 km, según el método de Ongley (Zăvoianu, 1985) y un ancho máximo de 2,45 $\mathrm{km}$. El factor de forma de 0,24 , confirma que la cuenca tiene una forma alargada (Zăvoianu, 1985). La longitud y el ancho promedio de la cuenca fueron 11,77 km y $0,86 \mathrm{~km}$, respectivamente, obtenidos del rectángulo equivalente.

\section{Análisis hipsométrico y frecuencia altimétrica}

Una cuenca hidrográfica es generalmente descrita por su integral hipsométrica $\int H b$ (Demoulin, 2010) un índice volumétrico propuesto por Strahler (1952) el cual vino a mejorar el método desarrollado por Langbein (Langbein, 1947). La integral hipsométrica $\int H b$, se puede definir como la integral de la distribución acumulada de la altitud relativa dentro de una cuenca hidrográfica. El análisis de frecuencia altimétrica se utiliza para describir las frecuencias de ciertos niveles de elevación y acompaña la descripción hipsométrica.

La subcuenca del río Toyogres muestra una curva hipsométrica de tipo cóncavo (fig. 4), donde el pico de la distribución altimétrica corresponde con las altitudes entre los 1300 y los $1540 \mathrm{~m}$. El valor de la integral hipsométrica, considerando el método propuesto por Pike y Wilson (1971), y corroborado a partir de la integración del área bajo la curva, es de $32 \%$. Este valor de la integral hipsométrica revela que la subcuenca del río Toyogres está iniciando su etapa de madurez, ya que valores menores del $30 \%$ son asociados a senetud y cercanos a $47 \%$ a madurez (Racca 2007). La elevación media de la cuenca es de 1600,48 m. 
Pablo Ramírez-Granados, Kevin Alfaro-Jiménez. Morphometry of Toyogres river sub-basin, Cartago, Costa Rica

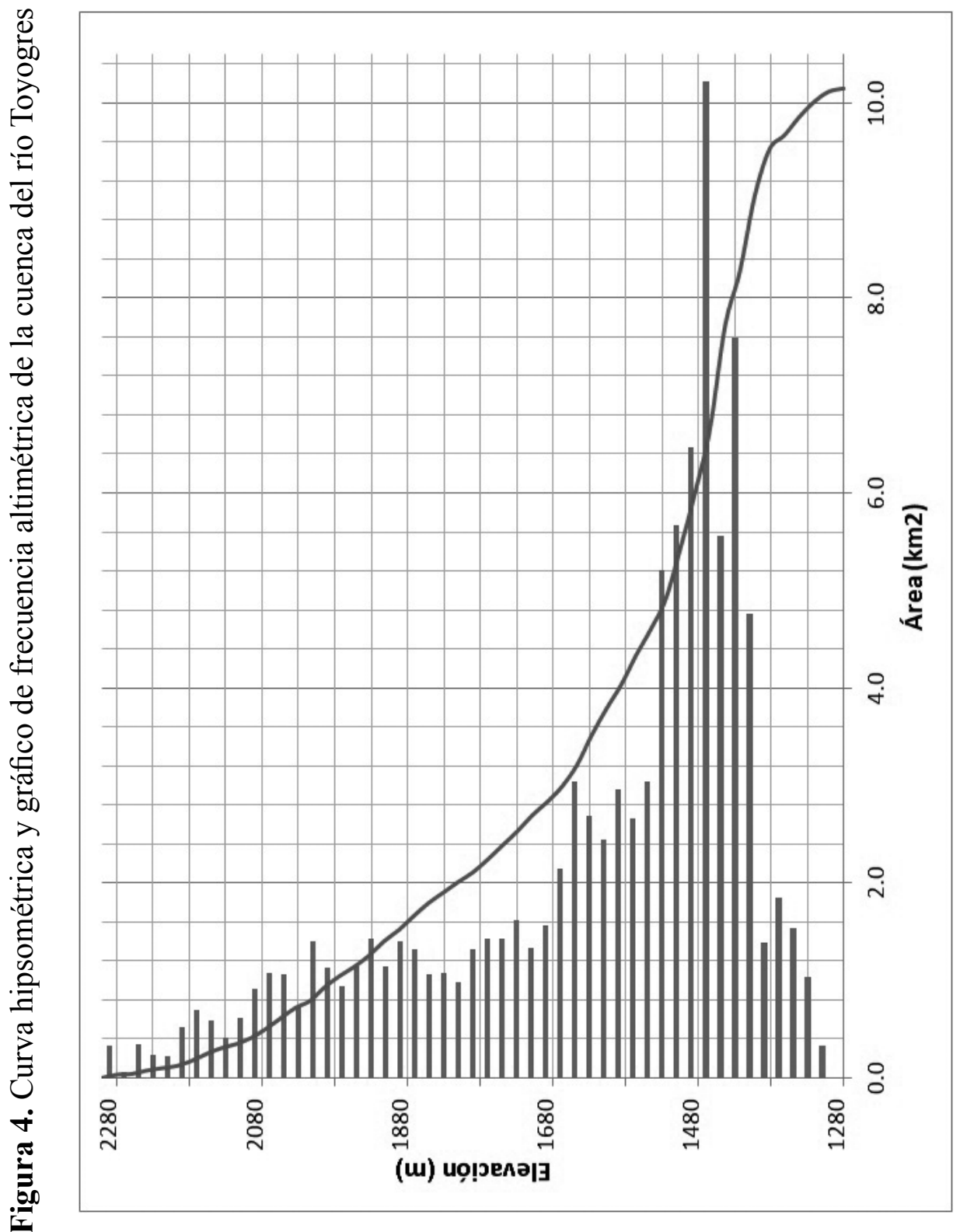


Pendiente de la cuenca.

La pendiente puede ser definida como la tangente del ángulo de inclinación de una línea o plano definido por la superficie (Zăvoianu, 1985). Existen varios métodos para calcular la pendiente promedio de la cuenca, algunos de ellos pueden ser consultados en Zăvoianu (1985), Chang et al., (1989), Villón (2004).

Al ser la cuenca hidrográfica, una superficie con una proyección horizontal irregular, resulta complicado establecer el ancho y el largo promedio. Para obtener ambos valores, es usado el criterio del rectángulo equivalente, donde se utiliza como punto de partida el área y el perímetro de la cuenca. El índice de pendiente (Ip) de la cuenca, según Villón (2004) es una ponderación que se establece entre las pendientes y el tramo recorrido por el río, tomando en cuenta el lado mayor del rectángulo equivalente. Para la cuenca, $I p$ arroja un valor de $26 \%$ o $14,6^{\circ}$ de pendiente promedio. El valor de la pendiente según el criterio de Alvord (Villón, 2004) fue de $14 \%$ o $8,21^{\circ}$. Usando el criterio del rectángulo equivalente, el valor de la pendiente para la cuenca del río Toyogres es de $8,5 \%$ o $4,85^{\circ}$. Tomando como base los segmentos del rectángulo equivalente, la pendiente con el criterio de Taylor \& Schwarz (Taylor y Schwarz 1952; Villón, 2004) resulta en un valor de $4,77 \%$ o $2,73^{\circ}$. Debido a las fuertes variaciones de las pendientes de una cuenca hidrográfica y a la imposibilidad de determinar, por la irregularidad de su forma, un valor más exacto, existe un criterio que puede ser usado como valor base para la pendiente de una cuenca.

Villón (2004) menciona que un criterio de naturaleza más real de medir la pendiente en una cuenca, es a partir de la compensación de áreas obtenidas de una recta de mejor ajuste, donde la condición es que dicha línea divida áreas iguales, por debajo y por encima de una línea de perfil longitudinal (fig. 5), esta última obtenida de un modelo digital del terreno (MDT). El valor de la pendiente, con el método de compensación de áreas, es de $14,1 \%$ o $8,02^{\circ}$. 


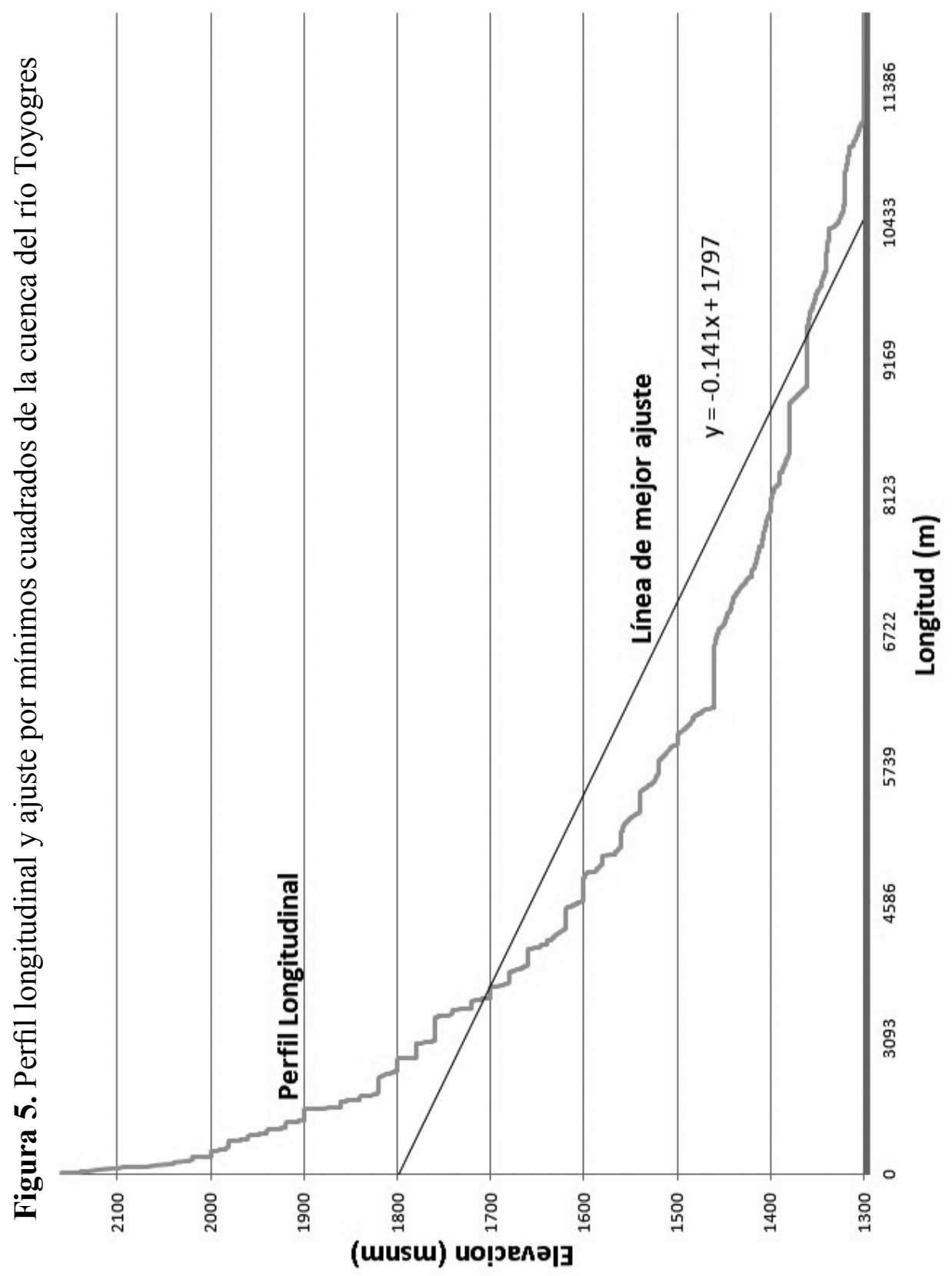


Este valor es coincidente con el criterio de Alvord y será el valor promedio de la pendiente de la cuenca del río Toyogres. Es importante mencionar que no se tomó en consideración el valor de la pendiente con el método de Taylor \& Schwarz, debido a la imposibilidad de dividir el perfil longitudinal en tramos irregulares (o en su defecto regulares) con contornos de escala 1:50 000. Esto debido a la escogencia de la geometría de la celda y a su digitalización.

\section{Drenajes}

El patrón de drenaje de una cuenca hidrográfica es el resultado de una combinación de factores: climático, tectónico, litológico, geomorfológico, de vegetación y de suelos (Esper Angillieri y Perucca, 2014).

La cuenca del río Toyogres manifiesta un patrón paralelo, con una dirección norte-sur. Considerando los contornos de $20 \mathrm{~m}$, muestra tres órdenes principales (fig. 6), obtenidos a partir del criterio de Strahler (Zăvoianu, 1985). 
Figura 6: Órdenes de corrientes de la cuenca del río Toyogres

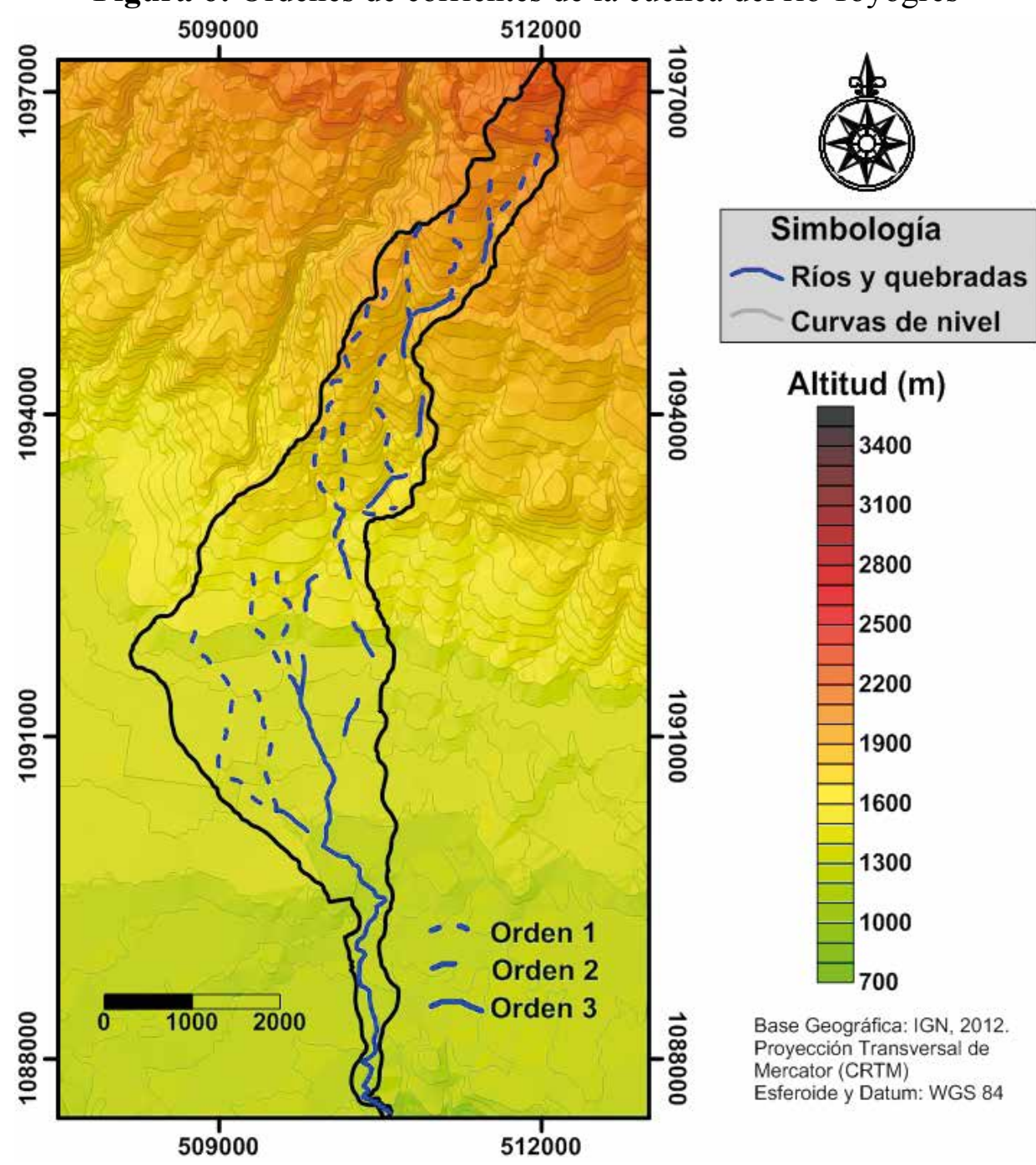

Los drenajes de orden 1 contabilizan un número de doce corrientes, con un promedio de $1,12 \mathrm{~km}$ y una longitud total de $13,54 \mathrm{~km}$ con una dirección de norte-noroeste a sureste. Los drenajes de orden 2 contabilizan siete corrientes, con una media de 1,27 km con una longitud total de 8,9 $\mathrm{km}$ y los drenajes de orden 3 contabilizan tres corrientes con una media de $1,62 \mathrm{~km}$ con una longitud total de 4,88 km (fig. 7). 
Pablo Ramírez-Granados, Kevin Alfaro-Jiménez. Morfometría de la subcuenca del río Toyogres, Cartago, Costa Rica

Figura 7: Relación número de corrientes y órdenes de corrientes.

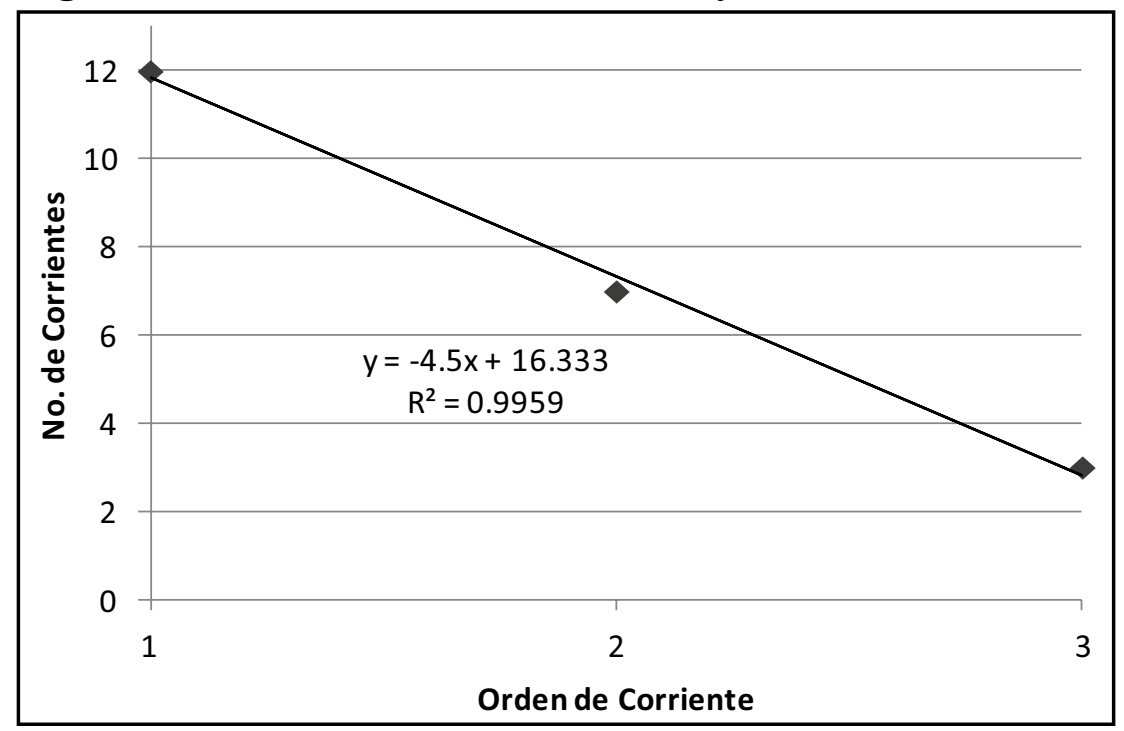

La longitud media de los drenajes fue de un total 1, $34 \mathrm{~km}$ y considerando cada orden, es notable que el valor de longitud media aumente conforme aumenta el número de orden (fig. 8).

Figura 8: Relación de la longitud media y los órdenes de corrientes.

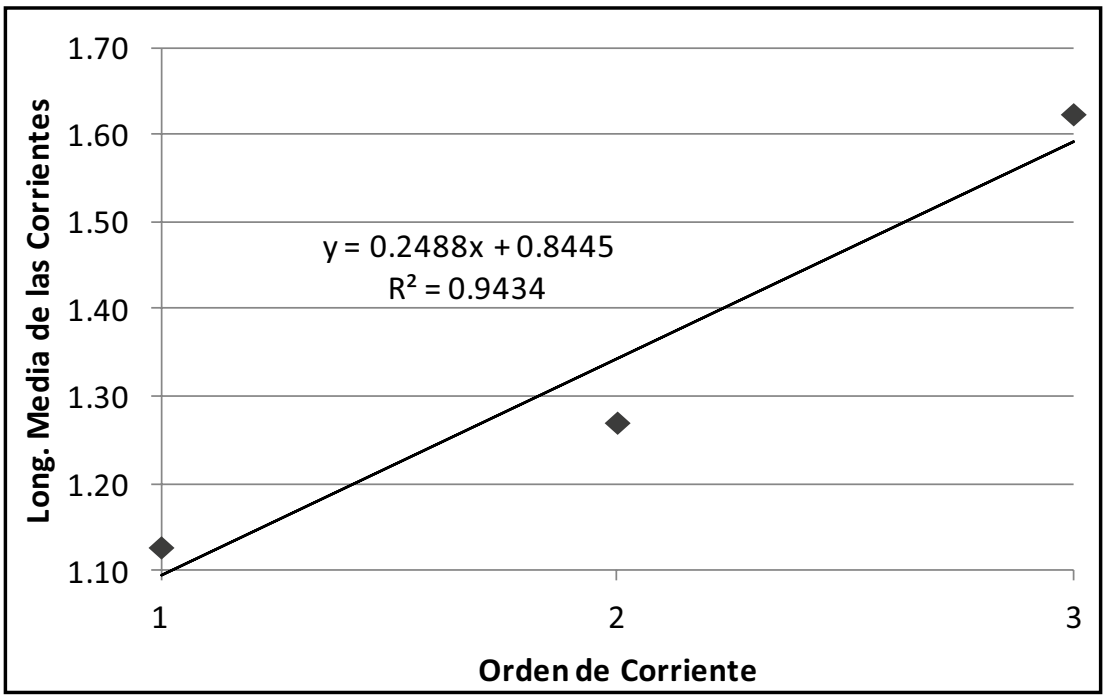


La razón de bifurcación $(R b)$ de la cuenca es de 2,02 (1, 71 para 1 y $2 ; 2,33$ para 2 y 3$)$ y su relación de longitudes de $1,20(1,13$ para 1 y 2 y 1,28 para 2 y 3 ).

La densidad de drenaje representa la cantidad de canales requerida para drenar una unidad de cuenca de drenaje (Gordon et al., 2004). La cuenca del río Toyogres exhibe una densidad de corrientes de $2,17 / \mathrm{km}^{2}$ y una densidad de drenajes de $2,69 \mathrm{~km} / \mathrm{km}^{2}$.

Vittala y Govindaiah (2004) indican que la densidad de drenaje varía entre 1,55 y $2,16 \mathrm{~km} / \mathrm{km}^{2}$, indicando una densidad de drenaje baja. Pareta y Pareta (2004) señalan que un valor de $2,79 \mathrm{~km} / \mathrm{km}^{2}$ se considera una densidad de drenaje moderada. Para la cuenca del río Toyogres el valor de la densidad de drenaje ronda entre bajo a moderado y podría ser un indicio de que la cuenca tiene un subsuelo permeable (Vittala y Govindaiah, 2004), lo cual podría relacionarse con los materiales volcánicos que lo conforman. Sin embargo, esta afirmación debe ser respaldada por pruebas de campo y de laboratorio.

\section{Conclusiones}

La subcuenca del río Toyogres parte del sistema de corrientes perennes que atraviesan la ciudad de Cartago y que corresponden con uno de los sistemas de drenaje del sector central de la ladera sur del volcán Irazú.

Está dominada por usos agrícolas y pastos en su parte alta y pastos y uso urbano en la parte baja. Los materiales geológicos que componen la subcuenca son de origen volcánico cuaternario, específicamente coluvios, lahares y aluviones, con algunos lentes de arcilla. Estos materiales son fácilmente observables, especialmente, en el sector de la cuenca media-baja, donde son reconocibles en el cauce principal y en algunos cortes de caminos.

Usando SIG y Microsoft Excel, se obtuvieron las propiedades geomorfométricas y morformétricas de la subcuenca. Esta se encuentra en el rango de los 1280 a los 2800 , con rangos de pendiente variables, desde los 0 a los $10^{\circ}$ en la parte baja y de 15 a $35^{\circ}$ en la parte media y alta. La orientación de la cuenca mostró direcciones de la pendiente en el sentido suroeste y sur y algunas pocas en la dirección sureste.

La subcuenca presenta un índice de compacidad de 2,22 y un factor de forma de 0,24, ambos índices confirman la forma alargada de la cuenca. La integral hipsométrica indica que es de tipo cóncavo, con un valor de un $32 \%$. La subcuenca presenta una pendiente de $14,1 \%$ a partir de su corriente principal. 
Los drenajes indican un patrón de tipo paralelo, con dirección nortesur y un máximo de 3 órdenes bajo el criterio de Strahler, siendo un máximo de orden 3. La relación de órdenes indica que a menor orden hay una mayor cantidad de corrientes y que al aumentar el orden, la longitud de las corrientes es mayor. La densidad de drenaje varía entre bajo a moderado y podría indicar que la subcuenca tiene un suelo permeable pero para comprobar esto, se deben de realizar más investigaciones.

\section{Referencias}

Alvarado, G. (1993). Volcanology and Petrology of Irazú Volcano, Costa Rica. (Tesis Ph.D.). University of Kiel.

Alvarado, G.,; Carr, M.,; Turrin, B., Swisher, C., Schmincke, H. \& Hudnut, K. (2006). Recent volcanic history of Irazú volcano, Costa Rica: Alternation and mixing of two magma batches and pervasive mixing. Geological Society of America Special Papers. 412, 259-276.

Alvarado, M., Durán, D., Fallas, K., Hernández, L. \& Valverde, R. (2006). Amenazas y vulnerabilidad: El caso de los ríos Reventado y Toyogres, Cartago. Revista Reflexiones. 85 (1-2), 1021-1209.

Avinash, K., Deepika, B. \& Jayappa, K. (2014). Basin geomorphology and drainage morphometry parameters used as indicators for groundwater prospect: Insight from Geographical Information System (GIS) technique. Journal of Earth Science. 25 (6), 1018-1032.

Boomfield, J; Bricker, S. \& Newell, A. (2011). Some relationships between lithology, basin form and hydrology: A case study from the Thames basin, UK. Hydrological Processes. 25, 2518-2530.

Brooks, K., Ffolliot, P. \& Magner, J. (2013). Hydrology and the Watershed Management. 4ta ed. Wiley-Blackwell.

Dade, W. (2001). Multiple scales in river basin morphology. American Journal of Science. 30, 60-73.

Demoulin, A. (2011). Basin and river profile morphometry: A new index with a high potential for relative dating of tectonic uplift. Geomorphology. 126, 97-107.

Esper Angillieri, M. Y. \& Perucca, L. (2014). Geomorphology and morphometry of the de La Fecha river basin, San Juan, Argentina. Environmental Earth Sciences. 72 (8), 3227-3237. 
Gordon, N., McMahon, T. \& Finlayson, B. (2004). Stream Ecology: An Introduction for Ecologist. 2da edición. Wiley.

Jordan, G. (2007). Digital Terrain Analysis on a GIS Environment. Concepts and Development in Peckham, R., Jordan, G. (Eds). Digital Terrain Modelling: Development and Application in a Policy Support Environment. Springer.

Krushensky, R. (1972). Geology of Istarú Quadrangle, Costa Rica. United States Geological Survey Bulletin. 1358.

Langbein, W. (1947). Topographic characteristic of drainage basins. Water-Supply Paper. 968-C. USGS.

Méndez-Morales, M. (2012). Calibración y validación del modelo hidorlógico SWMM en cuencas hidrográficas de alta pendiente en Costa Rica. Tecnología en Marcha. 26 (2), 20-32.

Mikhailova, M. (2015). Morphometry of river deltas. Water Resources. 42 (1), 52-62.

Prabu, P., Baskaran, R. (2013). Drainage morphometry of Upper Vaigai river sub-basin, Western Ghats, South India using remote sensing and GIS. Journal Geological Society of India. 82, 519-528.

Moore, I., Grayson, R. \& Ladson, A. (1991). Digital terrain modelling: A review of hydrological, geomorphological and biological applications. Hydrological Processes. 5, 3-30.

Newson, M. (1978). Drainage basin characteristics, their selection, derivation and analysis for a flood study of the British Isles. Earth Surface Processes. 3, 277-293.

Pareta, K. \& Pareta, U. (2011). Quantitative morphometric analysis of a waterhed of Yamuna basin, India using ASTER (DEM) data and GIS. International Journal of Geomatics and Geosciences. 2 (1), 248-269.

Pavanelli, N., Capaccioni, B., Sarocchi, D., Vaselli, O., Tassi, F. \& Duarte, E. (2004). Geology and stability of the southern flank of Irazú volcano, Costa Rica. Acta Vulcanológica. 16(1).

Pike, R. \& Wilson, S. (1971). Elevation-relief ratio, hypsometric integral, and geomorphic area-altitude analysis. Geological Society of America Bulletin. 82 (4), 1079-1084.

PRUGAM. (2008). Plan Regional Urbano de la Gram Área Metropolitana de Costa Rica 2008-2030. MIVAH-MOPT-MIDEPLAN-MINSALUD-INVU-IFAM-CNFL-AYA. 
Racca, J. (2007). Análisis hipsométrico, frecuencia altimétrica y pendientes medias a partir de modelos digitales del terreno. Boletín del Instituto de Fisiografía y Geología. 77 (1-2), 31-38.

Ramírez-Granados, P. \& Zúñiga-Mora, H. (2014). Modelado hidrogeológico para la caracterización de la hidrodinámica de las aguas subterráneas. Aplicación al acuifero de Cartago, Costa Rica. (Informe Final Proyecto 830-B2-039). Centro de Investigaciones en Ciencias Geológicas. Universidad de Costa Rica.

Shen, X., Vergara, H., Nikolopoulos, E., Anagnostou, E., Hong, Y., Hao, Z., Zhang, K., \& Mao, K. (2015). GDBC: A tool for generating global-scale distributed basin morphometry. Environmetal Modelling and Software. 83, 212-223.

Strahler, A. (1952). Hypsometric (area-altitude) analysis of erosional topography. Bulletin of the Geological Society of America. 53, 1117-1142.

Taylor, A. \& Schwarz, H. (1952). Unit-hidrograph lag and peak flow related to basin characteristic. Transactions American Geophysical Union. 33 (2), 235-246.

Villón, M. (2004). Hidrología. Editorial Tecnológica de Costa Rica.

Vittala, S., Givindaiah, S. \& Gowda, H. (2004). Morphometric analysis of sub-watersheds in the Pavagada area of Tumkur district, South India usinh remote sensing and GIS techniques. Journal of the Indian Society of Remote Sensing. 32 (4), 351-362.

Zaidi, F. (2011). Drainage basin morphometry for identifyng zones for artificial recharge: A case study from the Gagas River Basin, India. Journal Geological Society of India. 77, 160-166.

Zăvoianu, I. (1985). Morphometry of Drainage Basins. Developments in Water Science. 20. Elsevier. 